\title{
Shrinkage Testimator in Gamma Type-II Censored Data under LINEX Loss Function
}

\author{
Ali Shadrokh, Hassan Pazira \\ Department of statistics, Payame Nour University of Tehran, 19395-4697, Iran \\ Email: ali_shadrokh@pnu.ac.ir, pazira.b@gmail.com
}

Received September 1, 2012; revised January 1, 2013; accepted January 18, 2013

Copyright (c) 2013 Ali Shadrokh, Hassan Pazira. This is an open access article distributed under the Creative Commons Attribution License, which permits unrestricted use, distribution, and reproduction in any medium, provided the original work is properly cited.

\begin{abstract}
Prakash and Singh presented the shrinkage testimators under the invariant version of LINEX loss function for the scale parameter of an exponential distribution in presence Type-II censored data. In this paper, we extend this approach to gamma distribution, as Prakash and Singh's paper is a special case of this paper. In fact, some shrinkage testimators for the scale parameter of a gamma distribution, when Type-II censored data are available, have been suggested under the LINEX loss function assuming the shape parameter is to be known. The comparisons of the proposed testimators have been made with improved estimator. All these estimators are compared empirically using Monte Carlo simulation.
\end{abstract}

Keywords: Gamma Distribution; Shrinkage Estimator and Factor; Asymmetric Loss Function; Level of Significance; Testimation; Monte-Carlo Simulation

\section{Introduction}

In life-testing research, the most widely used life distribution is the Gamma with probability density function for any random variable $x$;

$$
f(x ; \lambda, \theta)=\frac{x^{\lambda-1}}{\Gamma(\lambda) \theta^{\lambda}} \mathrm{e}^{-\frac{x}{\theta}} ; x \geq 0, \lambda, \theta \geq 0 .
$$

Let $x_{1}, x_{2}, \cdots, x_{n}$ be the random samples of size $n$ taken form the Gamma distribution. The parameter $\lambda$ and $\theta$ are called the shape and scale parameter, respectively. It is crucial to have in-depth study of the (Classic and Bayes) estimate of the scale parameter of Gamma distribution because, in several cases, the distribution of the minimal sufficient statistics is Gamma (see Parsian and Kirmani [1]). Pazira and Shadrokh [2] derived Bayes estimators of the scale parameter of gamma distribution on the two asymmetric loss function LINEX and Precautionary by using several prior distributions and then compared the efficiency of all estimates. In the present paper, concentration is on the gamma distribution.

Ferguson [3], Zellner and Geisel [4], Aitchison and Dunsmore [5], Varian [6], and Berger [7] indicated to insufficient to symmetric loss function and just Varian [6] suggested asymmetric linear loss function. This loss function was widely used by several authors; among of them were Basu and Ebrahimi [8], Pandey [9], Soliman [10], and Prakash and Singh [11]. Following Basu and
Ebrahimi [8], the invariant form of the LINEX loss function (ILL) for any parameter $\theta$ is defined as

$$
L(\Delta)=\left\{\mathrm{e}^{c \Delta}-c \Delta-1\right\} ; c \neq 0, \Delta=\left(\frac{\hat{\theta}}{\theta}-1\right),
$$

where $c$ is the shape parameter and $\hat{\theta}$ is any estimate of the parameter $\theta$.

The LINEX loss function is convex and the shape of this loss function is determined by the value of $c$. The negative (positive) value of $c$ gives more weight to overestimation (underestimation) and its magnitude reflects the degree of asymmetry. It is seen that, for $c=1$, the function is quite asymmetric with overestimation being costlier than underestimation. If $c<0$, it rises almost exponentially when the estimation error $(\hat{\theta}-\theta)<0$ and almost linearly when $(\hat{\theta}-\theta)>0$. For small values of $|c|$, the LINEX loss function is almost symmetric and not far from squared error loss function.

Pandey [9], Parsian and Farsipour [12], Singh, Gupta, and Upadhyay [13], Misra and Meulen [14], Ahmadi, Doostparast, and Parsian [15], Xiao, Takada, and Shi [16], Singh, Prakash, and Singh [17] and others have used the LINEX loss function in the various estimation and prediction problems.

In life-testing, fatigue failures and other kinds of destructive test situations, the observations usually occurred in an ordered manner such a way that the weakest items 
failed first and then the second one and so on. Let us suppose that $n$ items are put on life test and terminate the experiment when $r(<n)$ items have failed. If $X_{(1)}, X_{(2)}, \cdots, X_{(r)}$ denote the first $r$ observations having a common density function as given in (1.1) then the joint probability density function is given by

$$
\begin{aligned}
& f\left(x_{(1)}, x_{(2)}, \cdots, x_{(r)} \mid \theta\right) \\
& =\frac{n !}{(n-r) !}\left(\frac{1}{\theta}\right)^{r \lambda}\left(\frac{1}{\Gamma(\lambda)}\right)^{r}\left(\prod_{i=1}^{r} x_{(i)}^{\lambda-1}\right) \mathrm{e}^{-\frac{T_{r}}{\theta}}
\end{aligned}
$$

where

$$
T_{r}=\sum_{i=1}^{r} x_{(i)}+(n-r) x_{(r)}
$$

$T_{r}$ is a complete sufficient statistic of $\theta$ and distributed as gamma distribution with parameters $(r \lambda, r \theta)$. The maximum likelihood estimator (MLE) of $\theta$ is given by

$$
\hat{\theta}=\frac{T_{r}}{r \lambda} \sim \frac{\theta}{2 r \lambda} \chi_{(2 r \lambda)}^{2}
$$

and can easily show that $\hat{\theta}$ is the minimum variance unbiased estimator (MVUE) of $\theta$.

Roa and Srivastava [18] considered a class for the total test time as

$$
Y=d \frac{T_{r}}{r \lambda},
$$

and found the value of the constant

$$
d=\frac{r \lambda}{c}\left(1-\mathrm{e}^{-\frac{c}{r \lambda+1}}\right)=d_{1}
$$

(say) which minimizes the risk of $Y$ under the ILL. The minimum risk estimator is

$$
Y_{1}=d_{1} \frac{T_{r}}{r \lambda}
$$

with the minimum risk under ILL

$$
\operatorname{Risk}\left(Y_{1}\right)=c+(r \lambda+1)\left(\mathrm{e}^{-\frac{c}{r \lambda+1}}-1\right),
$$

for $\lambda=1$, see Prakash and Singh [11].

In the present paper, some shrinkage testimators for the scale parameter of a gamma distribution, when Type-II censored data are available, have been suggested under the ILL loss function assuming the shape parameter is to be known.

\section{Shrinkage Testimators and their Properties}

Following Thompson [19], the shrinkage estimator for the parameter $\theta$ is given by

$$
\hat{Y}=\theta_{0}+k\left(\frac{T_{r}}{r \lambda}-\theta_{0}\right), 0 \leq k \leq 1 .
$$

The value of the shrinkage factor $k$ near to the zero implies strong belief in the guess value $\theta_{0}$ and near to one implies a strong belief in the sample values. Several researchers have studied the performance of the shrinkage estimators and found that the shrinkage estimator performs better with respect to any usual estimator when the guess value $\theta_{0}$ is close to the parameter $\theta$. This suggests that we may test the hypothesis $H_{0}: \theta=\theta_{0}$ against $H_{1}: \theta \neq \theta_{0}$. A test statistic

$$
\frac{2 T_{r}}{\theta_{0}} \sim \chi_{(2 r \lambda)}^{2}
$$

is available for testing the hypothesis $H_{0}$.

The loss for estimator $\hat{Y}$ under the ILL is defined as

$$
L(\hat{Y})=\left\{\mathrm{e}^{c \Delta}-c \Delta-1\right\},
$$

where

$$
c \Delta=c\left\{\delta-1+k\left(T_{r} /(r \lambda \theta)-\delta\right)\right\}
$$

and

$$
\delta=\frac{\theta_{0}}{\theta} .
$$

The risk of the proposed shrinkage estimator $\hat{Y}$ under the ILL is given by

$$
\operatorname{Risk}(\hat{Y})=\mathrm{e}^{c(\delta-1)} \mathrm{e}^{-c k \delta}\left(1-\frac{c k}{r \lambda}\right)^{-r \lambda}-c(\delta-1)(1-k)-1
$$

The value of $k=k_{\min }$ (say), which minimizes the risk Risk $(\hat{Y})$ is thus obtained by solving the given Equation

$$
(1-\delta) \mathrm{e}^{c(1-\delta)}=\mathrm{e}^{-c k \delta}\left(1-\frac{c k}{r \lambda}\right)^{-(r \lambda+1)}\left\{1-\delta\left(1-\frac{c k}{r \lambda}\right)\right\} .
$$

The value of $k_{\min }$ depends upon the unknown parameter $\theta$. Hence, an estimate $\hat{k}$ of $k_{\min }$ is obtained by replacing the parameter $\theta$ to its minimum variance unbiased estimator. Based on this, the proposed shrinkage testimator for the scale parameter $\theta$ is defined as

$$
\hat{\theta}_{1}=d_{1} \frac{T_{r}}{r \lambda}+\left((1-\hat{k}) \theta_{0}+\left(\hat{k}-d_{1}\right) \frac{T_{r}}{r \lambda}\right) I_{\left(t_{1} \leq T_{r} \leq t_{2}\right)},(2.4
$$

where $I_{(A)}$ denotes the indicator of $A, t_{1}=m_{1} \theta_{0} / 2$ and $t_{2}=m_{2} \theta_{0} / 2$. Here $m_{1}$ and $m_{2}$ are the values of the lower and upper

$$
100 \frac{\alpha}{2} \%
$$


points of the chi-square distribution with $2 r \lambda$ degrees of freedom. The risk under the ILL for the shrinkage testimator $\hat{\theta}_{1}$ is given by

$$
\begin{aligned}
& \operatorname{Risk}\left(\hat{\theta}_{1}\right) \\
& =\left\{\mathrm{e}^{c(\delta-1)} G\left(w_{1}, w_{2}, \mathrm{e}^{f}\right)-\mathrm{e}^{-c} G\left(w_{1}, w_{2}, f_{0}\right)\right. \\
& \quad-G\left(w_{1}, w_{2}, f\right)-c \delta G\left(w_{1}, w_{2}, 1\right)+G\left(w_{1}, w_{2}, f_{0}\right), \\
& \left.\quad+c+(1+r \lambda)\left(\exp \left(-\frac{c}{r \lambda+1}\right)-1\right)\right\}
\end{aligned}
$$

where

$$
\begin{gathered}
w_{1}=\delta m_{1} / 2, \quad w_{2}=\delta m_{2} / 2, f=c \hat{k}(w / r \lambda-\delta), \\
f_{0}=\left(w c d_{1} / r \lambda\right), \quad G(u, v, y)=\frac{1}{\Gamma(r \lambda)} \int_{u}^{v} y w^{r \lambda-1} \mathrm{e}^{-w} \mathrm{~d} w
\end{gathered}
$$

and $y$ may be a function of $w$. For $\lambda=1$, see Prakash and Singh [11].

Waikar, Schuurmann, and Raghunathan [20] has suggested an idea of selecting the shrinkage factor which is the function of the test statistic i.e., under $H_{0}: \theta=\theta_{0}$

$$
\begin{aligned}
& m_{1} \leq \frac{2 T_{r}}{\theta_{0}} \leq m_{2} \\
& \Leftrightarrow 0 \leq k_{1} \text { (say) }=\frac{1}{m_{2}-m_{1}}\left(\frac{2 T_{r}}{\theta_{0}}-m_{1}\right) \leq 1
\end{aligned}
$$

Therefore, the proposed shrinkage testimator based on $k_{1}$ is given by

$$
\hat{\theta}_{2}=d_{1} \frac{T_{r}}{r \lambda}+\left(\left(1-k_{1}\right) \theta_{0}+\left(k_{1}-d_{1}\right) \frac{T_{r}}{r \lambda}\right) I_{\left(t_{1} \leq T_{r} \leq t_{2}\right)} \text {. }
$$

The risk under the ILL for the shrinkage testimator $\hat{\theta}_{2}$ is given by

$$
\begin{aligned}
& \operatorname{Risk}\left(\hat{\theta}_{2}\right) \\
& =\left\{\mathrm{e}^{c(\delta-1)} G\left(w_{1}, w_{2}, \mathrm{e}^{f_{1}}\right)-\mathrm{e}^{-c} G\left(w_{1}, w_{2}, f_{0}\right)\right. \\
& \quad-G\left(w_{1}, w_{2}, f_{1}\right)-c \delta G\left(w_{1}, w_{2}, 1\right)+G\left(w_{1}, w_{2}, f_{0}\right), \\
& \left.\quad+c+(1+r \lambda)\left(\exp \left(-\frac{c}{r \lambda+1}\right)-1\right)\right\}
\end{aligned}
$$

where

$$
f_{1}=\frac{c}{m_{2}-m_{1}}\left(\frac{2 w}{\delta}-m_{1}\right)\left(\frac{w}{r \lambda}-\delta\right) .
$$

For $\lambda=1$, see Prakash and Singh [11].

When $H_{0}: \theta=\theta_{0}$ is accepted,

$$
m_{1} \leq 2 r \lambda \leq m_{2} \Rightarrow m_{1} / 2 r \lambda \leq 1 .
$$

If one is interested in taking smaller values of the shrinkage factor, he can take $m_{1} / 2 r \lambda \cong 1$. The proposed shrinkage testimator is

$$
\hat{\theta}_{3}=d_{1} \frac{T_{r}}{r \lambda}+\left(\left(1-k_{2}\right) \theta_{0}+\left(k_{2}-d_{1}\right) \frac{T_{r}}{r \lambda}\right) I_{\left(t_{1} \leq T_{r} \leq t_{2}\right)}
$$

where

$$
k_{2}=\frac{2 r \lambda}{m_{2}-m_{1}}\left|\frac{T_{r}}{r \lambda \theta_{0}}-1\right|
$$

it may be possible that the value of shrinkage factor is negative so positive is taken. Adke, Waikar, and Schuurmann [21] and Pandey, Malik, and Srivastava [22] have considered this type of shrinkage factor. The risk of the shrinkage testimator $\hat{\theta}_{3}$ is given by

$$
\begin{aligned}
& \operatorname{Risk}\left(\hat{\theta}_{3}\right) \\
= & \left\{\mathrm{e}^{c(\delta-1)} G\left(w_{1}, w_{2}, \mathrm{e}^{f_{2}}\right)-\mathrm{e}^{-c} G\left(w_{1}, w_{2}, f_{0}\right)\right. \\
& -G\left(w_{1}, w_{2}, f_{2}\right)-c \delta G\left(w_{1}, w_{2}, 1\right)+G\left(w_{1}, w_{2}, f_{0}\right), \\
& \left.+c+(1+r \lambda)\left(\exp \left(-\frac{c}{r \lambda+1}\right)-1\right)\right\}
\end{aligned}
$$

where

$$
f_{2}=\frac{c}{m_{2}-m_{1}}\left|\frac{2 w}{\delta}-2 r \lambda\right|\left(\frac{w}{r \lambda}-\delta\right) .
$$

For $\lambda=1$, see Prakash and Singh [11].

The minimum value of constant $d, d_{1}$ obtained for the class $Y=d T_{r} / r \lambda$, lies between zero and one. Hence, it may be a choice for the shrinkage factor. Thus, the proposed shrinkage testimator may be considered as

$$
\hat{\theta}_{4}=d_{1} \frac{T_{r}}{r \lambda}+\left(\left(1-d_{1}\right) \theta_{0}\right) I_{\left(t_{1} \leq T_{r} \leq t_{2}\right)}
$$

The risk of the proposed shrinkage testimator $\hat{\theta}_{4}$ under ILL is given by

$$
\begin{aligned}
& \operatorname{Risk}\left(\hat{\theta}_{4}\right)=\left\{\mathrm{e}^{c(\delta-1)} G\left(w_{1}, w_{2}, \mathrm{e}^{f_{1}}\right)\right. \\
& -\mathrm{e}^{-c} G\left(w_{1}, w_{2}, f_{0}\right)-c \delta\left(1+d_{1}\right) G\left(w_{1}, w_{2}, 1\right), \\
& \left.+c+(1+r \lambda)\left(\exp \left(-\frac{c}{r \lambda+1}\right)-1\right)\right\}
\end{aligned}
$$

where

$$
f_{3}=\frac{c d_{1}}{m_{2}-m_{1}}\left(\frac{w}{r \lambda}-\delta\right) .
$$

For $\lambda=1$, see Prakash and Singh [11].

\section{Numerical Illustration}

The relative efficiency for $\hat{\theta}_{i} ; i=1, \cdots, 4$, with respect to the minimum risk improved estimator under the ILL is defined as 


$$
\operatorname{RE}\left(\hat{\theta}_{i}, Y_{1}\right)=\frac{\operatorname{Risk}\left(Y_{1}\right)}{\operatorname{Risk}\left(\hat{\theta}_{i}\right)} ; i=1, \cdots, 4
$$

The expression for the relative efficiency $R E\left(\hat{\theta}_{i}, Y_{1}\right)$; $i=1, \cdots, 4$, is the function of $r, c, \delta, \lambda$ and $\alpha$. For the selected values of $r=6,8,10 ; c=0.25,0.5,1,1.5 ; \delta=$ $0.4(0.2) 1.8 ; \alpha=0.01,0.05,0.1$ and $\lambda=0.5,2.5$, the relative efficiencies have been calculated and presented in Tables 1-8. Only positive values of $c$ are considered because overestimation in mean life is more serious

Table 1. $R E\left(\hat{\theta}_{1}, Y_{1}\right)$ when $\lambda=0.5$.

\begin{tabular}{|c|c|c|c|c|c|c|c|c|c|}
\hline \multirow{2}{*}{$c$} & \multirow{2}{*}{$\alpha$} & \multicolumn{8}{|c|}{$\delta$} \\
\hline & & 0.4 & 0.6 & 0.8 & 1.0 & 1.2 & 1.4 & 1.6 & 1.8 \\
\hline \multicolumn{10}{|c|}{$r=6$} \\
\hline \multirow{4}{*}{0.25} & 0.01 & 1.0116 & 1.0100 & 0.9997 & 0.9997 & 0.9997 & 0.9997 & 0.9997 & 0.9997 \\
\hline & 0.05 & 1.0138 & 1.0110 & 1.0102 & 1.0100 & 1.0101 & 1.0102 & 1.0103 & 1.0106 \\
\hline & 0.10 & 1.0158 & 1.0120 & 1.0108 & 1.0105 & 1.0106 & 1.0108 & 1.0112 & 1.0116 \\
\hline & 0.01 & 1.0463 & 1.0442 & 1.0446 & 1.0451 & 1.0449 & 1.0444 & 1.0439 & 1.0436 \\
\hline \multirow[t]{3}{*}{0.50} & 0.05 & 1.0508 & 1.0463 & 1.0459 & 1.0464 & 1.0466 & 1.0466 & 1.0465 & 1.0468 \\
\hline & 0.10 & 1.0552 & 1.0492 & 1.0481 & 1.0486 & 1.0491 & 1.0496 & 1.0500 & 1.0508 \\
\hline & 0.01 & 1.1600 & 1.1840 & 1.2050 & 1.2150 & 1.2090 & 1.1930 & 1.1780 & 1.1680 \\
\hline \multirow[t]{2}{*}{1.00} & 0.05 & 1.1570 & 1.1790 & 1.2030 & 1.2180 & 1.2150 & 1.2000 & 1.1840 & 1.1720 \\
\hline & 0.10 & 1.1610 & 1.1810 & 1.2070 & 1.2250 & 1.2240 & 1.2090 & 1.1920 & 1.1790 \\
\hline \multirow[t]{4}{*}{1.50} & 0.05 & 1.2660 & 1.3480 & 1.4470 & 1.5160 & 1.4830 & 1.3950 & 1.3200 & 1.2700 \\
\hline & 0.10 & 1.2640 & 1.3400 & 1.4440 & 1.5230 & 1.4960 & 1.4070 & 1.3270 & 1.2720 \\
\hline & $r=8$ & & & & & & & & \\
\hline & 0.01 & 1.0100 & 0.9982 & 0.9978 & 0.9978 & 0.9978 & 0.9978 & 0.9978 & 0.9979 \\
\hline \multirow[t]{3}{*}{0.25} & 0.05 & 1.0124 & 0.9991 & 0.9983 & 0.9981 & 0.9982 & 0.9983 & 0.9985 & 0.9988 \\
\hline & 0.10 & 1.0144 & 1.0101 & 0.9989 & 0.9986 & 0.9987 & 0.9990 & 0.9994 & 1.0100 \\
\hline & 0.01 & 1.0383 & 1.0358 & 1.0359 & 1.0362 & 1.0361 & 1.0358 & 1.0356 & 1.0356 \\
\hline \multirow[t]{3}{*}{0.50} & 0.05 & 1.0432 & 1.0383 & 1.0375 & 1.0379 & 1.0382 & 1.0384 & 1.0387 & 1.0393 \\
\hline & 0.10 & 1.0481 & 1.0415 & 1.0400 & 1.0404 & 1.0410 & 1.0417 & 1.0426 & 1.0438 \\
\hline & 0.01 & 1.1250 & 1.1460 & 1.1630 & 1.1710 & 1.1660 & 1.1560 & 1.1460 & 1.1400 \\
\hline \multirow[t]{2}{*}{1.00} & 0.05 & 1.1260 & 1.1450 & 1.1660 & 1.1800 & 1.1780 & 1.1660 & 1.1550 & 1.1480 \\
\hline & 0.01 & 1.2210 & 1.2970 & 1.3650 & 1.4040 & 1.3740 & 1.3150 & 1.2690 & 1.2410 \\
\hline \multirow[t]{4}{*}{1.50} & 0.05 & 1.2090 & 1.2790 & 1.3660 & 1.4280 & 1.4000 & 1.3310 & 1.2760 & 1.2420 \\
\hline & 0.10 & 1.2140 & 1.2820 & 1.3810 & 1.4620 & 1.4360 & 1.3540 & 1.2890 & 1.2480 \\
\hline & $r=10$ & & & & & & & & \\
\hline & 0.01 & 0.9990 & 0.9969 & 0.9966 & 0.9965 & 0.9965 & 0.9966 & 0.9966 & 0.9967 \\
\hline \multirow[t]{3}{*}{0.25} & 0.05 & 1.0115 & 0.9980 & 0.9970 & 0.9969 & 0.9969 & 0.9971 & 0.9974 & 0.9977 \\
\hline & 0.10 & 1.0138 & 0.9990 & 0.9976 & 0.9973 & 0.9975 & 0.9978 & 0.9983 & 0.9990 \\
\hline & 0.01 & 1.0331 & 1.0302 & 1.0301 & 1.0304 & 1.0303 & 1.0302 & 1.0301 & 1.0303 \\
\hline \multirow[t]{3}{*}{0.50} & 0.05 & 1.0386 & 1.0332 & 1.0322 & 1.0325 & 1.0328 & 1.0331 & 1.0337 & 1.0346 \\
\hline & 0.10 & 1.0442 & 1.0369 & 1.0350 & 1.0353 & 1.0360 & 1.0370 & 1.0382 & 1.0398 \\
\hline & 0.01 & 1.1030 & 1.1210 & 1.1360 & 1.1430 & 1.1390 & 1.1310 & 1.1250 & 1.1220 \\
\hline \multirow{3}{*}{1.00} & 0.05 & 1.1070 & 1.1240 & 1.1450 & 1.1590 & 1.1560 & 1.1460 & 1.1380 & 1.1330 \\
\hline & 0.10 & 1.1170 & 1.1340 & 1.1590 & 1.1800 & 1.1790 & 1.1660 & 1.1540 & 1.1460 \\
\hline & 0.01 & 1.1770 & 1.2440 & 1.3030 & 1.3350 & 1.3110 & 1.2670 & 1.2350 & 1.2170 \\
\hline \multirow[t]{2}{*}{1.50} & 0.05 & 1.1750 & 1.2380 & 1.3230 & 1.3840 & 1.3570 & 1.2950 & 1.2510 & 1.2240 \\
\hline & 0.10 & 1.1860 & 1.2500 & 1.3570 & 1.4510 & 1.4190 & 1.3330 & 1.2710 & 1.2350 \\
\hline
\end{tabular}


Table 2. $R E\left(\hat{\theta}_{2}, Y_{1}\right)$ when $\lambda=0.5$.

\begin{tabular}{|c|c|c|c|c|c|c|c|c|c|}
\hline \multirow{2}{*}{$c$} & \multirow{2}{*}{$\alpha$} & \multicolumn{8}{|c|}{$\delta$} \\
\hline & & 0.4 & 0.6 & 0.8 & 1.0 & 1.2 & 1.4 & 1.6 & 1.8 \\
\hline \multicolumn{10}{|c|}{$r=6$} \\
\hline & 0.01 & 1.0116 & 1.0100 & 0.9997 & 0.9996 & 0.9996 & 0.0096 & 0.9996 & 0.9996 \\
\hline \multirow[t]{3}{*}{0.25} & 0.05 & 1.0139 & 1.0110 & 1.0102 & 1.0100 & 1.0100 & 1.0101 & 1.0103 & 1.0104 \\
\hline & 0.10 & 1.0158 & 1.0120 & 1.0108 & 1.0105 & 1.0106 & 1.0108 & 1.0111 & 1.0114 \\
\hline & 0.01 & 1.0465 & 1.0441 & 1.0440 & 1.0441 & 1.0441 & 1.0435 & 1.0425 & 1.0408 \\
\hline \multirow[t]{3}{*}{0.50} & 0.05 & 1.0510 & 1.0465 & 1.0456 & 1.0456 & 1.0457 & 1.0455 & 1.0449 & 1.0438 \\
\hline & 0.10 & 1.0555 & 1.0494 & 1.0479 & 1.0479 & 1.0483 & 1.0484 & 1.0483 & 1.0476 \\
\hline & 0.01 & 1.1630 & 1.1810 & 1.1890 & 1.1910 & 1.1860 & 1.1730 & 1.1530 & 1.1270 \\
\hline \multirow[t]{3}{*}{1.00} & 0.05 & 1.1590 & 1.1800 & 1.1950 & 1.1980 & 1.1910 & 1.1770 & 1.1560 & 1.1320 \\
\hline & 0.10 & 1.1630 & 1.1830 & 1.2030 & 1.2100 & 1.2030 & 1.1860 & 1.1640 & 1.1390 \\
\hline & 0.01 & 1.2980 & 1.3640 & 1.3760 & 1.3640 & 1.3370 & 1.2900 & 1.2280 & 1.1660 \\
\hline \multirow[t]{4}{*}{1.50} & 0.05 & 1.2730 & 1.3550 & 1.4050 & 1.3970 & 1.3510 & 1.2900 & 1.2250 & 1.1680 \\
\hline & 0.10 & 1.2700 & 1.3500 & 1.4240 & 1.4330 & 1.3810 & 1.3060 & 1.2340 & 1.1750 \\
\hline & $r=8$ & & & & & & & & \\
\hline & 0.01 & 1.0100 & 0.9982 & 0.9978 & 0.9977 & 0.9977 & 0.9978 & 0.9978 & 0.9978 \\
\hline \multirow[t]{3}{*}{0.25} & 0.05 & 1.0124 & 0.9992 & 0.9983 & 0.9981 & 0.9981 & 0.9983 & 0.9985 & 0.9987 \\
\hline & 0.10 & 1.0145 & 1.0102 & 0.9989 & 0.9986 & 0.9987 & 0.9989 & 0.9993 & 0.9998 \\
\hline & 0.01 & 1.0384 & 1.0357 & 1.0355 & 1.0357 & 1.0356 & 1.0353 & 1.0344 & 1.0332 \\
\hline \multirow[t]{3}{*}{0.50} & 0.05 & 1.0434 & 1.0384 & 1.0373 & 1.0374 & 1.0376 & 1.0377 & 1.0374 & 1.0367 \\
\hline & 0.10 & 1.0483 & 1.0416 & 1.0399 & 1.0399 & 1.0405 & 1.0409 & 1.0411 & 1.0410 \\
\hline & 0.01 & 1.1270 & 1.1450 & 1.1540 & 1.1570 & 1.1540 & 1.1440 & 1.1260 & 1.1050 \\
\hline \multirow[t]{3}{*}{1.00} & 0.05 & 1.1270 & 1.1460 & 1.1620 & 1.1680 & 1.1640 & 1.1510 & 1.1330 & 1.1120 \\
\hline & 0.10 & 1.1340 & 1.1530 & 1.1730 & 1.1830 & 1.1790 & 1.1640 & 1.1430 & 1.1210 \\
\hline & 0.01 & 1.2260 & 1.2920 & 1.3180 & 1.3190 & 1.3010 & 1.2580 & 1.1990 & 1.1430 \\
\hline \multirow[t]{2}{*}{1.50} & 0.05 & 1.2130 & 1.2850 & 1.3450 & 1.3530 & 1.3200 & 1.2650 & 1.2030 & 1.1490 \\
\hline & 0.10 & 1.2170 & 1.2890 & 1.3710 & 1.3970 & 1.3550 & 1.2840 & 1.2150 & 1.1580 \\
\hline \multicolumn{10}{|c|}{$r=10$} \\
\hline \multirow{3}{*}{0.25} & 0.01 & 0.9990 & 0.9969 & 0.9965 & 0.9965 & 0.9965 & 0.9965 & 0.9966 & 0.9966 \\
\hline & 0.05 & 1.0116 & 0.9980 & 0.9970 & 0.9968 & 0.9969 & 0.9971 & 0.9973 & 0.9976 \\
\hline & 0.10 & 1.0138 & 0.9990 & 0.9976 & 0.9973 & 0.9974 & 0.9978 & 0.9982 & 0.9988 \\
\hline & 0.01 & 1.0332 & 1.0302 & 1.0299 & 1.0300 & 1.0300 & 1.0297 & 1.0291 & 1.0281 \\
\hline \multirow[t]{3}{*}{0.50} & 0.05 & 1.0387 & 1.0333 & 1.0321 & 1.0321 & 1.0324 & 1.0326 & 1.0325 & 1.0322 \\
\hline & 0.10 & 1.0443 & 1.0370 & 1.0350 & 1.0350 & 1.0356 & 1.0363 & 1.0369 & 1.0371 \\
\hline & 0.01 & 1.1040 & 1.1210 & 1.1310 & 1.1340 & 1.1320 & 1.1230 & 1.1080 & 1.0895 \\
\hline \multirow[t]{3}{*}{1.00} & 0.05 & 1.1080 & 1.1260 & 1.1430 & 1.1500 & 1.1460 & 1.1350 & 1.1180 & 1.0987 \\
\hline & 0.10 & 1.1170 & 1.1360 & 1.1590 & 1.1710 & 1.1670 & 1.1520 & 1.1320 & 1.1110 \\
\hline & 0.01 & 1.1800 & 1.2430 & 1.2750 & 1.2810 & 1.2670 & 1.2280 & 1.1740 & 1.1240 \\
\hline \multirow[t]{2}{*}{1.50} & 0.05 & 1.1770 & 1.2430 & 1.3100 & 1.3270 & 1.2990 & 1.2450 & 1.1840 & 1.1330 \\
\hline & 0.10 & 1.1880 & 1.2570 & 1.3520 & 1.3930 & 1.3510 & 1.2730 & 1.2010 & 1.1460 \\
\hline
\end{tabular}


Table 3. $R E\left(\hat{\theta}_{3}, Y_{1}\right)$ when $\lambda=0.5$.

\begin{tabular}{|c|c|c|c|c|c|c|c|c|c|}
\hline \multirow{2}{*}{$c$} & \multirow{2}{*}{$\alpha$} & \multicolumn{8}{|c|}{$\delta$} \\
\hline & & 0.4 & 0.6 & 0.8 & 1.0 & 1.2 & 1.4 & 1.6 & 1.8 \\
\hline \multicolumn{10}{|c|}{$r=6$} \\
\hline & 0.01 & 1.0116 & 1.0100 & 0.9997 & 0.9997 & 0.9997 & 0.9997 & 0.9997 & 0.9997 \\
\hline \multirow[t]{3}{*}{0.25} & 0.05 & 1.0138 & 1.0110 & 1.0102 & 1.0100 & 1.0100 & 1.0102 & 1.0103 & 1.0105 \\
\hline & 0.10 & 1.0158 & 1.0120 & 1.0108 & 1.0105 & 1.0106 & 1.0108 & 1.0112 & 1.0115 \\
\hline & 0.01 & 1.0462 & 1.0442 & 1.0444 & 1.0447 & 1.0447 & 1.0442 & 1.0433 & 1.0419 \\
\hline \multirow[t]{3}{*}{0.50} & 0.05 & 1.0507 & 1.0464 & 1.0458 & 1.0461 & 1.0464 & 1.0464 & 1.0460 & 1.0453 \\
\hline & 0.10 & 1.0552 & 1.0493 & 1.0480 & 1.0483 & 1.0489 & 1.0494 & 1.0496 & 1.0494 \\
\hline & 0.01 & 1.1590 & 1.1840 & 1.2000 & 1.2060 & 1.2020 & 1.1890 & 1.1670 & 1.1430 \\
\hline \multirow[t]{3}{*}{1.00} & 0.05 & 1.1560 & 1.1790 & 1.2010 & 1.2110 & 1.2090 & 1.1960 & 1.1750 & 1.1530 \\
\hline & 0.10 & 1.1600 & 1.1810 & 1.2050 & 1.2200 & 1.2190 & 1.2060 & 1.1850 & 1.1620 \\
\hline & 0.01 & 1.2880 & 1.3770 & 1.4380 & 1.4590 & 1.4330 & 1.3660 & 1.2830 & 1.2080 \\
\hline \multirow[t]{4}{*}{1.50} & 0.05 & 1.2650 & 1.3500 & 1.4360 & 1.4740 & 1.4460 & 1.3750 & 1.2930 & 1.2220 \\
\hline & 0.10 & 1.2640 & 1.3420 & 1.4380 & 1.4920 & 1.4680 & 1.3910 & 1.3050 & 1.2330 \\
\hline & $r=8$ & & & & & & & & \\
\hline & 0.01 & 1.0100 & 0.9982 & 0.9978 & 0.9977 & 0.9978 & 0.9978 & 0.9978 & 0.9978 \\
\hline \multirow[t]{3}{*}{0.25} & 0.05 & 1.0124 & 0.9991 & 0.9983 & 0.9981 & 0.9982 & 0.9983 & 0.9985 & 0.9988 \\
\hline & 0.10 & 1.0144 & 1.0101 & 0.9989 & 0.9986 & 0.9987 & 0.9990 & 0.9994 & 0.9999 \\
\hline & 0.01 & 1.0382 & 1.0357 & 1.0358 & 1.0360 & 1.0360 & 1.0356 & 1.0350 & 1.0340 \\
\hline \multirow[t]{3}{*}{0.50} & 0.05 & 1.0431 & 1.0383 & 1.0375 & 1.0377 & 1.0381 & 1.0382 & 1.0381 & 1.0379 \\
\hline & 0.10 & 1.0481 & 1.0415 & 1.0399 & 1.0402 & 1.0409 & 1.0416 & 1.0421 & 1.0424 \\
\hline & 0.01 & 1.1240 & 1.1450 & 1.1600 & 1.1660 & 1.1630 & 1.1520 & 1.1360 & 1.1170 \\
\hline \multirow[t]{3}{*}{1.00} & 0.05 & 1.1250 & 1.1450 & 1.1660 & 1.1760 & 1.1750 & 1.1630 & 1.1460 & 1.1280 \\
\hline & 0.10 & 1.1320 & 1.1510 & 1.1750 & 1.1900 & 1.1900 & 1.1780 & 1.1590 & 1.1390 \\
\hline & 0.01 & 1.2180 & 1.2940 & 1.3530 & 1.3740 & 1.3550 & 1.3010 & 1.2330 & 1.1730 \\
\hline \multirow[t]{4}{*}{1.50} & 0.05 & 1.2080 & 1.2800 & 1.3620 & 1.4030 & 1.3810 & 1.3190 & 1.2490 & 1.1890 \\
\hline & 0.10 & 1.2130 & 1.2820 & 1.3790 & 1.4400 & 1.4190 & 1.3440 & 1.2650 & 1.2020 \\
\hline & $r=10$ & & & & & & & & \\
\hline & 0.01 & 0.9990 & 0.9969 & 0.9966 & 0.9965 & 0.9965 & 0.9965 & 0.9966 & 0.9966 \\
\hline \multirow[t]{3}{*}{0.25} & 0.05 & 1.0115 & 0.9980 & 0.9970 & 0.9968 & 0.9969 & 0.9971 & 0.9973 & 0.9976 \\
\hline & 0.10 & 1.0138 & 0.9990 & 0.9976 & 0.9973 & 0.9974 & 0.9978 & 0.9983 & 0.9989 \\
\hline & 0.01 & 1.0330 & 1.0302 & 1.0301 & 1.0303 & 1.0303 & 1.0300 & 1.0295 & 1.0289 \\
\hline \multirow[t]{3}{*}{0.50} & 0.05 & 1.0385 & 1.0332 & 1.0322 & 1.0324 & 1.0327 & 1.0330 & 1.0331 & 1.0332 \\
\hline & 0.10 & 1.0441 & 1.0369 & 1.0350 & 1.0352 & 1.0360 & 1.0368 & 1.0376 & 1.0384 \\
\hline & 0.01 & 1.1020 & 1.1210 & 1.1350 & 1.1400 & 1.1380 & 1.1290 & 1.1150 & 1.0993 \\
\hline \multirow[t]{3}{*}{1.00} & 0.05 & 1.1060 & 1.1240 & 1.1450 & 1.1560 & 1.1540 & 1.1440 & 1.1280 & 1.1120 \\
\hline & 0.10 & 1.1160 & 1.1340 & 1.1590 & 1.1770 & 1.1770 & 1.1630 & 1.1450 & 1.1260 \\
\hline & 0.01 & 1.1740 & 1.2420 & 1.2970 & 1.3180 & 1.3020 & 1.2550 & 1.1990 & 1.1490 \\
\hline \multirow[t]{2}{*}{1.50} & 0.05 & 1.1730 & 1.2380 & 1.3210 & 1.3660 & 1.3450 & 1.2850 & 1.2200 & 1.1660 \\
\hline & 0.10 & 1.1850 & 1.2500 & 1.3560 & 1.4320 & 1.4060 & 1.3230 & 1.2430 & 1.1830 \\
\hline
\end{tabular}


Table 4. $R E\left(\hat{\theta}_{4}, Y_{1}\right)$ when $\lambda=0.5$.

\begin{tabular}{|c|c|c|c|c|c|c|c|c|c|}
\hline \multirow{2}{*}{$c$} & \multirow{2}{*}{$\alpha$} & \multicolumn{8}{|c|}{$\delta$} \\
\hline & & 0.4 & 0.6 & 0.8 & 1.0 & 1.2 & 1.4 & 1.6 & 1.8 \\
\hline \multicolumn{10}{|c|}{$r=6$} \\
\hline & 0.01 & 1.0178 & 1.0157 & 1.0164 & 1.0178 & 1.0195 & 1.0215 & 1.0237 & 1.0263 \\
\hline \multirow[t]{3}{*}{0.25} & 0.05 & 1.0225 & 1.0175 & 1.0172 & 1.0184 & 1.0204 & 1.0229 & 1.0260 & 1.0297 \\
\hline & 0.10 & 1.0266 & 1.0193 & 1.0183 & 1.0193 & 1.0215 & 1.0246 & 1.0287 & 1.0338 \\
\hline & 0.01 & 1.1420 & 1.2100 & 1.6020 & 1.8800 & 1.2840 & 1.1820 & 1.1410 & 1.1220 \\
\hline \multirow[t]{3}{*}{0.50} & 0.05 & 1.1650 & 1.2070 & 1.5070 & 2.0600 & 1.2900 & 1.1830 & 1.1430 & 1.1240 \\
\hline & 0.10 & 1.1860 & 1.2130 & 1.4710 & 2.3000 & 1.3030 & 1.1890 & 1.1480 & 1.1310 \\
\hline & 0.01 & 2.3100 & 1.3070 & 1.1890 & 1.1470 & 1.1270 & 1.1180 & 1.1160 & 1.1230 \\
\hline \multirow[t]{3}{*}{1.00} & 0.05 & 5.4400 & 1.3900 & 1.2090 & 1.1550 & 1.1330 & 1.1230 & 1.1220 & 1.1310 \\
\hline & 0.10 & 28.100 & 1.4760 & 1.2320 & 1.1660 & 1.1400 & 1.1310 & 1.1320 & 1.1430 \\
\hline & 0.01 & 1.9600 & 1.3330 & 1.2220 & 1.1810 & 1.1630 & 1.1590 & 1.1720 & 1.2200 \\
\hline \multirow[t]{4}{*}{1.50} & 0.05 & 2.9300 & 1.4100 & 1.2430 & 1.1900 & 1.1700 & 1.1670 & 1.1820 & 1.2350 \\
\hline & 0.10 & 5.1900 & 1.4910 & 1.2680 & 1.2030 & 1.1800 & 1.1780 & 1.1980 & 1.2590 \\
\hline & $r=8$ & & & & & & & & \\
\hline & 0.01 & 1.0163 & 1.0134 & 1.0138 & 1.0151 & 1.0168 & 1.0189 & 1.0214 & 1.0243 \\
\hline \multirow[t]{3}{*}{0.25} & 0.05 & 1.0218 & 1.0152 & 1.0146 & 1.0157 & 1.0177 & 1.0204 & 1.0239 & 1.0285 \\
\hline & 0.10 & 1.0266 & 1.0170 & 1.0155 & 1.0165 & 1.0188 & 1.0222 & 1.0269 & 1.0336 \\
\hline & 0.01 & 1.1410 & 1.2280 & 5.4400 & 1.2850 & 1.1500 & 1.1070 & 1.0872 & 1.0767 \\
\hline \multirow[t]{3}{*}{0.50} & 0.05 & 1.1700 & 1.2200 & 2.3900 & 1.3110 & 1.1530 & 1.1090 & 1.0904 & 1.0812 \\
\hline & 0.10 & 1.1950 & 1.2250 & 1.9890 & 1.3430 & 1.1600 & 1.1140 & 1.0956 & 1.0879 \\
\hline & 0.01 & 1.8350 & 1.2150 & 1.1340 & 1.1060 & 1.0918 & 1.0851 & 1.0834 & 1.0869 \\
\hline \multirow[t]{3}{*}{1.00} & 0.05 & 3.1800 & 1.2740 & 1.1480 & 1.1110 & 1.0956 & 1.0895 & 1.0894 & 1.0954 \\
\hline & 0.10 & 8.3200 & 1.3340 & 1.1640 & 1.1180 & 1.1010 & 1.0955 & 1.0974 & 1.1070 \\
\hline & 0.01 & 1.7190 & 1.2430 & 1.1620 & 1.1320 & 1.1190 & 1.1160 & 1.1230 & 1.1510 \\
\hline \multirow[t]{2}{*}{1.50} & 0.05 & 2.4700 & 1.3030 & 1.1770 & 1.1390 & 1.1240 & 1.1220 & 1.1330 & 1.1670 \\
\hline & 0.10 & 4.0100 & 1.3640 & 1.1950 & 1.1480 & 1.1320 & 1.1310 & 1.1460 & 1.1880 \\
\hline \multicolumn{10}{|c|}{$r=10$} \\
\hline \multirow{3}{*}{0.25} & 0.01 & 1.0156 & 1.0118 & 1.0120 & 1.0132 & 1.0149 & 1.0169 & 1.0195 & 1.0228 \\
\hline & 0.05 & 1.0220 & 1.0136 & 1.0127 & 1.0137 & 1.0157 & 1.0185 & 1.0224 & 1.0278 \\
\hline & 0.10 & 1.0276 & 1.0154 & 1.0136 & 1.0144 & 1.0167 & 1.0203 & 1.0258 & 1.0339 \\
\hline \multirow{3}{*}{0.50} & 0.01 & 1.1410 & 1.2490 & 1.8260 & 1.1700 & 1.1020 & 1.0760 & 1.0633 & 1.0564 \\
\hline & 0.05 & 1.1780 & 1.2340 & 2.9000 & 1.1820 & 1.1040 & 1.0785 & 1.0669 & 1.0616 \\
\hline & 0.10 & 1.2090 & 1.2370 & 11.100 & 1.1970 & 1.1090 & 1.0825 & 1.0721 & 1.0687 \\
\hline \multirow{3}{*}{1.00} & 0.01 & 1.6690 & 1.1670 & 1.1040 & 1.0822 & 1.0718 & 1.0666 & 1.0653 & 1.0678 \\
\hline & 0.05 & 2.7200 & 1.2160 & 1.1150 & 1.0862 & 1.0748 & 1.0706 & 1.0712 & 1.0767 \\
\hline & 0.10 & 5.4900 & 1.2650 & 1.1270 & 1.0917 & 1.0793 & 1.0761 & 1.0789 & 1.0881 \\
\hline \multirow{3}{*}{1.50} & 0.01 & 1.6170 & 1.1930 & 1.1270 & 1.1040 & 1.0938 & 1.0911 & 1.0964 & 1.1160 \\
\hline & 0.05 & 2.3300 & 1.2450 & 1.1400 & 1.1090 & 1.0982 & 1.0972 & 1.1060 & 1.1320 \\
\hline & 0.10 & 3.8900 & 1.2970 & 1.1540 & 1.1160 & 1.1040 & 1.1050 & 1.1180 & 1.1540 \\
\hline
\end{tabular}


Table 5. $R E\left(\hat{\theta}_{1}, Y_{1}\right)$ when $\lambda=2.5$.

\begin{tabular}{|c|c|c|c|c|c|c|c|c|c|}
\hline \multirow{2}{*}{$c$} & \multirow{2}{*}{$\alpha$} & \multicolumn{8}{|c|}{$\delta$} \\
\hline & & 0.4 & 0.6 & 0.8 & 1.0 & 1.2 & 1.4 & 1.6 & 1.8 \\
\hline \multicolumn{10}{|c|}{$r=6$} \\
\hline & 0.01 & 0.9980 & 0.9936 & 0.9928 & 0.9927 & 0.9927 & 0.9928 & 0.9930 & 0.9934 \\
\hline \multirow[t]{3}{*}{0.25} & 0.05 & 1.0150 & 0.9954 & 0.9934 & 0.9931 & 0.9932 & 0.9936 & 0.9945 & 0.9958 \\
\hline & 0.10 & 1.0235 & 0.9972 & 0.9939 & 0.9934 & 0.9937 & 0.9945 & 0.9961 & 0.9988 \\
\hline & 0.01 & 1.0209 & 1.0155 & 1.0142 & 1.0142 & 1.0143 & 1.0146 & 1.0153 & 1.0164 \\
\hline \multirow[t]{3}{*}{0.50} & 0.05 & 1.0370 & 1.0244 & 1.0197 & 1.0194 & 1.0202 & 1.0221 & 1.0252 & 1.0291 \\
\hline & 0.10 & 1.0568 & 1.0342 & 1.0247 & 1.0239 & 1.0260 & 1.0304 & 1.0372 & 1.0452 \\
\hline & 0.01 & 1.0478 & 1.0620 & 1.0838 & 1.0938 & 1.0901 & 1.0849 & 1.0801 & 1.0743 \\
\hline \multirow[t]{3}{*}{1.00} & 0.05 & 1.0804 & 1.1060 & 1.2190 & 1.4390 & 1.3670 & 1.2500 & 1.1790 & 1.1350 \\
\hline & 0.10 & 1.1200 & 1.1610 & 1.6850 & 1.7500 & 1.1300 & 2.2000 & 1.3500 & 1.2070 \\
\hline & 0.01 & 1.0735 & 1.1240 & 1.2470 & 1.3370 & 1.2840 & 1.2270 & 1.1870 & 1.1520 \\
\hline \multirow[t]{4}{*}{1.50} & 0.05 & 1.1200 & 1.2210 & 3.1800 & 1.4120 & 1.5970 & 3.4200 & 1.5400 & 1.2600 \\
\hline & 0.10 & 1.1750 & 1.3530 & 1.4610 & 1.2170 & 1.2690 & 1.6350 & 2.4000 & 1.3610 \\
\hline & $r=8$ & & & & & & & & \\
\hline & 0.01 & 1.9997 & 0.9934 & 0.9923 & 0.9922 & 0.9922 & 0.9924 & 0.9927 & 0.9934 \\
\hline \multirow[t]{3}{*}{0.25} & 0.05 & 1.0227 & 0.9958 & 0.9929 & 0.9925 & 0.9927 & 0.9933 & 0.9945 & 0.9970 \\
\hline & 0.10 & 1.0405 & 0.9983 & 0.9933 & 0.9927 & 0.9931 & 0.9942 & 0.9967 & 0.0119 \\
\hline & 0.01 & 1.0229 & 1.0154 & 1.0131 & 1.0131 & 1.0132 & 1.0138 & 1.0151 & 0.0170 \\
\hline \multirow[t]{3}{*}{0.50} & 0.05 & 1.0513 & 1.0289 & 1.0196 & 1.0189 & 1.0202 & 1.0238 & 1.0300 & 0.0381 \\
\hline & 0.10 & 1.0898 & 1.0437 & 1.0248 & 1.0230 & 1.0263 & 1.0345 & 1.0490 & 0.0674 \\
\hline & 0.01 & 1.0491 & 1.0676 & 1.1230 & 1.1610 & 1.1470 & 1.1280 & 1.1070 & 0.0863 \\
\hline \multirow[t]{3}{*}{1.00} & 0.05 & 1.1050 & 1.1560 & 2.3000 & 1.2370 & 1.2910 & 1.8060 & 1.5920 & 0.2120 \\
\hline & 0.10 & 1.1760 & 1.2790 & 1.3180 & 1.1360 & 1.1550 & 1.2870 & 3.3900 & 0.3670 \\
\hline & 0.01 & 1.0739 & 1.1440 & 3.3800 & 1.8200 & 3.1300 & 2.2000 & 1.3700 & 0.1900 \\
\hline \multirow[t]{2}{*}{1.50} & 0.05 & 1.1530 & 1.3900 & 1.2040 & 1.1260 & 1.1480 & 1.2460 & 2.2400 & 0.4300 \\
\hline & 0.10 & 1.2480 & 1.8240 & 1.1670 & 1.1030 & 1.1210 & 1.2090 & 1.8510 & 0.6260 \\
\hline \multicolumn{10}{|c|}{$r=10$} \\
\hline \multirow{3}{*}{0.25} & 0.01 & 1.0129 & 0.9935 & 0.9920 & 0.9919 & 0.9919 & 0.9921 & 0.9927 & 0.9937 \\
\hline & 0.05 & 1.0376 & 0.9967 & 0.9925 & 0.9921 & 0.9923 & 0.9931 & 0.9950 & 0.9993 \\
\hline & 0.10 & 1.0752 & 1.0100 & 0.9929 & 0.9923 & 0.9927 & 0.9940 & 0.9977 & 1.0175 \\
\hline \multirow{3}{*}{0.50} & 0.01 & 1.0284 & 1.0172 & 1.0131 & 1.0129 & 1.0132 & 1.0142 & 1.0166 & 1.0201 \\
\hline & 0.05 & 1.0797 & 1.0369 & 1.0199 & 1.0184 & 1.0204 & 1.0265 & 1.0385 & 1.0559 \\
\hline & 0.10 & 1.1540 & 1.0586 & 1.0246 & 1.0216 & 1.0260 & 1.0392 & 1.0675 & 1.1090 \\
\hline \multirow{3}{*}{1.00} & 0.01 & 1.0584 & 1.0881 & 1.5470 & 2.2200 & 3.0100 & 1.6490 & 1.2300 & 1.1220 \\
\hline & 0.05 & 1.1540 & 1.2800 & 1.1670 & 1.0926 & 1.1040 & 1.1640 & 1.6570 & 1.4010 \\
\hline & 0.10 & 1.2770 & 1.5740 & 1.1450 & 1.0784 & 1.0894 & 1.1500 & 1.5380 & 1.7050 \\
\hline \multirow{3}{*}{1.50} & 0.01 & 1.0865 & 1.2150 & 1.2080 & 1.1400 & 1.1620 & 1.2400 & 2.1100 & 1.3070 \\
\hline & 0.05 & 1.2180 & 2.2000 & 1.1090 & 1.0740 & 1.0851 & 1.1300 & 1.3540 & 1.8710 \\
\hline & 0.10 & 1.3700 & 6.3400 & 1.1090 & 1.0692 & 1.0822 & 1.1410 & 1.4590 & 2.0800 \\
\hline
\end{tabular}


Table 6. $R E\left(\hat{\theta}_{2}, Y_{1}\right)$ when $\lambda=2.5$.

\begin{tabular}{|c|c|c|c|c|c|c|c|c|c|}
\hline \multirow{2}{*}{$c$} & \multirow{2}{*}{$\alpha$} & \multicolumn{8}{|c|}{$\delta$} \\
\hline & & 0.4 & 0.6 & 0.8 & 1.0 & 1.2 & 1.4 & 1.6 & 1.8 \\
\hline \multicolumn{10}{|c|}{$r=6$} \\
\hline & 0.01 & 0.9980 & 0.9936 & 0.9928 & 0.9927 & 0.9927 & 0.9928 & 0.9930 & 0.9934 \\
\hline \multirow[t]{3}{*}{0.25} & 0.05 & 1.0150 & 0.9954 & 0.9934 & 0.9931 & 0.9932 & 0.9936 & 0.9944 & 0.9957 \\
\hline & 0.10 & 1.0235 & 0.9972 & 0.9939 & 0.9934 & 0.9937 & 0.9945 & 0.9960 & 0.9986 \\
\hline & 0.01 & 1.0209 & 1.0155 & 1.0142 & 1.0141 & 1.0142 & 1.0144 & 1.0145 & 1.0148 \\
\hline \multirow[t]{3}{*}{0.50} & 0.05 & 1.0370 & 1.0244 & 1.0197 & 1.0193 & 1.0201 & 1.0216 & 1.0236 & 1.0261 \\
\hline & 0.10 & 1.0568 & 1.0342 & 1.0248 & 1.0238 & 1.0258 & 1.0296 & 1.0347 & 1.0407 \\
\hline & 0.01 & 1.0478 & 1.0622 & 1.0831 & 1.0907 & 1.0878 & 1.0760 & 1.0606 & 1.0475 \\
\hline \multirow[t]{3}{*}{1.00} & 0.05 & 1.0804 & 1.1070 & 1.2210 & 1.3860 & 1.3280 & 1.1920 & 1.1170 & 1.0836 \\
\hline & 0.10 & 1.1200 & 1.1630 & 1.7200 & 1.9140 & 2.7300 & 1.5400 & 1.1990 & 1.1280 \\
\hline & 0.01 & 1.0736 & 1.1250 & 1.2400 & 1.2970 & 1.2600 & 1.1700 & 1.1010 & 1.0645 \\
\hline \multirow[t]{4}{*}{1.50} & 0.05 & 1.1200 & 1.2250 & 3.0700 & 1.4780 & 1.7590 & 1.7780 & 1.1920 & 1.1080 \\
\hline & 0.10 & 1.1750 & 1.3610 & 1.4470 & 1.2300 & 1.2960 & 3.1300 & 1.3130 & 1.1590 \\
\hline & $r=8$ & & & & & & & & \\
\hline & 0.01 & 1.9997 & 0.9934 & 0.9923 & 0.9922 & 0.9922 & 1.9924 & 0.9927 & 0.9933 \\
\hline \multirow[t]{3}{*}{0.25} & 0.05 & 1.0227 & 0.9958 & 0.9929 & 0.9925 & 0.9927 & 1.9933 & 0.9945 & 0.9968 \\
\hline & 0.10 & 1.0405 & 0.9983 & 0.9933 & 0.9927 & 0.9931 & 1.9942 & 0.9966 & 0.0116 \\
\hline & 0.01 & 1.0229 & 1.0155 & 1.0131 & 1.0130 & 1.0132 & 1.0135 & 1.0141 & 1.0152 \\
\hline \multirow[t]{3}{*}{0.50} & 0.05 & 1.0513 & 1.0289 & 1.0196 & 1.0188 & 1.0201 & 1.0230 & 1.0276 & 1.0336 \\
\hline & 0.10 & 1.0898 & 1.0438 & 1.0249 & 1.0229 & 1.0261 & 1.0332 & 1.0447 & 1.0595 \\
\hline & 0.01 & 1.0491 & 1.0678 & 1.1220 & 1.1520 & 1.1400 & 1.1020 & 1.0692 & 1.0501 \\
\hline \multirow[t]{3}{*}{1.00} & 0.05 & 1.1050 & 1.1570 & 2.2000 & 1.2560 & 1.3250 & 3.9100 & 1.2000 & 1.1130 \\
\hline & 0.10 & 1.1760 & 1.2820 & 1.3090 & 1.1400 & 1.1640 & 1.4480 & 1.4680 & 1.1970 \\
\hline & 0.01 & 1.0739 & 1.1450 & 3.0300 & 2.1900 & 8.6200 & 1.3430 & 1.1180 & 1.0661 \\
\hline \multirow[t]{2}{*}{1.50} & 0.05 & 1.1530 & 1.3990 & 1.2020 & 1.1310 & 1.1570 & 1.4250 & 1.3410 & 1.1380 \\
\hline & 0.10 & 1.2480 & 1.8530 & 1.1650 & 1.1050 & 1.1270 & 1.2930 & 1.7330 & 1.2260 \\
\hline \multicolumn{10}{|c|}{$r=10$} \\
\hline \multirow{3}{*}{0.25} & 0.01 & 1.0129 & 1.9935 & 1.9920 & 1.9919 & 1.9919 & 1.9921 & 1.9926 & 1.9936 \\
\hline & 0.05 & 1.0376 & 1.9967 & 1.9925 & 1.9921 & 1.9923 & 1.9931 & 1.9949 & 1.9991 \\
\hline & 0.10 & 1.0752 & 1.0100 & 1.9929 & 1.9923 & 1.9927 & 1.9940 & 1.9976 & 1.0171 \\
\hline \multirow{3}{*}{0.50} & 0.01 & 1.0284 & 1.0172 & 1.0131 & 1.0129 & 1.0131 & 1.0138 & 1.0153 & 1.0176 \\
\hline & 0.05 & 1.0797 & 1.0370 & 1.0199 & 1.0183 & 1.0203 & 1.0253 & 1.0346 & 1.0483 \\
\hline & 0.10 & 1.1540 & 1.0587 & 1.0246 & 1.0215 & 1.0257 & 1.0372 & 1.0601 & 1.0947 \\
\hline \multirow{3}{*}{1.00} & 0.01 & 1.0584 & 1.0884 & 1.5360 & 3.1200 & 3.9100 & 1.2490 & 1.0981 & 1.0610 \\
\hline & 0.05 & 1.1540 & 1.2830 & 1.1650 & 1.0952 & 1.1090 & 1.2380 & 1.5580 & 1.1730 \\
\hline & 0.10 & 1.2770 & 1.5820 & 1.1430 & 1.0799 & 1.0925 & 1.1910 & 5.7400 & 1.3220 \\
\hline \multirow{3}{*}{1.50} & 0.01 & 1.0866 & 1.2170 & 1.2090 & 1.1480 & 1.1740 & 1.6640 & 1.1760 & 1.0775 \\
\hline & 0.05 & 1.2180 & 2.2500 & 1.1080 & 1.0758 & 1.0884 & 1.1770 & 2.0700 & 1.1970 \\
\hline & 0.10 & 1.3700 & 7.2000 & 1.1080 & 1.0703 & 1.0850 & 1.1810 & 6.4600 & 1.3390 \\
\hline
\end{tabular}


Table 7. $R E\left(\hat{\theta}_{3}, Y_{1}\right)$ when $\lambda=2.5$.

\begin{tabular}{|c|c|c|c|c|c|c|c|c|c|}
\hline \multirow{2}{*}{$c$} & \multirow{2}{*}{$\alpha$} & \multicolumn{8}{|c|}{$\delta$} \\
\hline & & 0.4 & 0.6 & 0.8 & 1.0 & 1.2 & 1.4 & 1.6 & 1.8 \\
\hline \multicolumn{10}{|c|}{$r=6$} \\
\hline & 0.01 & 0.9980 & 0.9936 & 0.9928 & 0.9927 & 0.9927 & 0.9928 & 0.9930 & 0.9934 \\
\hline \multirow[t]{3}{*}{0.25} & 0.05 & 1.0150 & 0.9954 & 0.9934 & 0.9931 & 0.9932 & 0.9936 & 0.9944 & 0.9957 \\
\hline & 0.10 & 1.0235 & 0.9972 & 0.9939 & 0.9934 & 0.9937 & 0.9945 & 0.9960 & 0.9987 \\
\hline & 0.01 & 1.0208 & 1.0154 & 1.0142 & 1.0142 & 1.0143 & 1.0145 & 1.0148 & 1.0155 \\
\hline \multirow[t]{3}{*}{0.50} & 0.05 & 1.0370 & 1.0243 & 1.0197 & 1.0194 & 1.0202 & 1.0218 & 1.0243 & 1.0273 \\
\hline & 0.10 & 1.0567 & 1.0340 & 1.0247 & 1.0239 & 1.0260 & 1.0300 & 1.0357 & 1.0425 \\
\hline & 0.01 & 1.0475 & 1.0609 & 1.0836 & 1.0929 & 1.0896 & 1.0792 & 1.0669 & 1.0562 \\
\hline \multirow[t]{3}{*}{1.00} & 0.05 & 1.0801 & 1.1040 & 1.2190 & 1.4210 & 1.3610 & 1.2160 & 1.1370 & 1.1010 \\
\hline & 0.10 & 1.1200 & 1.1580 & 1.6840 & 1.7970 & 2.1700 & 1.7560 & 1.2450 & 1.1540 \\
\hline & 0.01 & 1.0730 & 1.1200 & 1.2440 & 1.3260 & 1.2790 & 1.1890 & 1.1240 & 1.0865 \\
\hline \multirow[t]{4}{*}{1.50} & 0.05 & 1.1200 & 1.2140 & 3.2200 & 1.4290 & 1.6140 & 2.5700 & 1.2670 & 1.1470 \\
\hline & 0.10 & 1.1750 & 1.3420 & 1.4610 & 1.2210 & 1.2710 & 1.9470 & 1.4780 & 1.2120 \\
\hline & & & & & & & & & \\
\hline & 0.01 & 0.9997 & 0.9934 & 0.9923 & 0.9922 & 0.9922 & 0.9924 & 0.9927 & 0.9933 \\
\hline \multirow[t]{3}{*}{0.25} & 0.05 & 1.0226 & 0.9958 & 0.9929 & 0.9925 & 0.9927 & 0.9933 & 0.9945 & 0.9969 \\
\hline & 0.10 & 1.0405 & 0.9983 & 0.9933 & 0.9927 & 0.9931 & 0.9942 & 0.9966 & 1.0117 \\
\hline & 0.01 & 1.0229 & 1.0154 & 1.0131 & 1.0131 & 1.0132 & 1.0136 & 1.0145 & 1.0160 \\
\hline \multirow[t]{3}{*}{0.50} & 0.05 & 1.0513 & 1.0287 & 1.0196 & 1.0188 & 1.0202 & 1.0233 & 1.0285 & 1.0355 \\
\hline & 0.10 & 1.0897 & 1.0435 & 1.0248 & 1.0230 & 1.0263 & 1.0338 & 1.0464 & 1.0627 \\
\hline & 0.01 & 1.0489 & 1.0659 & 1.1220 & 1.1580 & 1.1450 & 1.1110 & 1.0810 & 1.0619 \\
\hline \multirow[t]{3}{*}{1.00} & 0.05 & 1.1050 & 1.1510 & 2.3500 & 1.2430 & 1.2970 & 3.7600 & 1.2800 & 1.1430 \\
\hline & 0.10 & 1.1760 & 1.2700 & 1.3200 & 1.1370 & 1.1570 & 1.3550 & 1.8480 & 1.2480 \\
\hline & 0.01 & 1.0736 & 1.1370 & 3.0600 & 1.8940 & 3.7500 & 1.4730 & 1.1660 & 1.0953 \\
\hline \multirow[t]{2}{*}{1.50} & 0.05 & 1.1520 & 1.3650 & 1.2050 & 1.1280 & 1.1500 & 1.3200 & 1.7700 & 1.2020 \\
\hline & 0.10 & 1.2470 & 1.7580 & 1.1680 & 1.1040 & 1.1220 & 1.2460 & 1.9000 & 1.3190 \\
\hline \multicolumn{10}{|c|}{$r=10$} \\
\hline \multirow{3}{*}{0.25} & 0.01 & 1.0129 & 0.9935 & 0.9920 & 0.9919 & 0.9919 & 0.9921 & 0.9926 & 0.9937 \\
\hline & 0.05 & 1.0375 & 0.9967 & 0.9925 & 0.9921 & 0.9923 & 0.9931 & 0.9949 & 0.9992 \\
\hline & 0.10 & 1.0752 & 1.0100 & 0.9929 & 0.9923 & 0.9927 & 0.9940 & 0.9977 & 0.0173 \\
\hline \multirow{3}{*}{0.50} & 0.01 & 1.0284 & 1.0171 & 1.0131 & 1.0129 & 1.0131 & 1.0140 & 1.0158 & 1.0187 \\
\hline & 0.05 & 1.0797 & 1.0367 & 1.0198 & 1.0184 & 1.0204 & 1.0258 & 1.0362 & 1.0515 \\
\hline & 0.10 & 1.1540 & 1.0582 & 1.0245 & 1.0216 & 1.0259 & 1.0381 & 1.0631 & 1.1010 \\
\hline \multirow{3}{*}{1.00} & 0.01 & 1.0583 & 1.0852 & 1.5180 & 2.3800 & 8.9500 & 1.3270 & 1.1300 & 1.0799 \\
\hline & 0.05 & 1.1540 & 1.2660 & 1.1690 & 1.0934 & 1.1060 & 1.2000 & 3.9100 & 1.2340 \\
\hline & 0.10 & 1.2770 & 1.5400 & 1.1460 & 1.0789 & 1.0900 & 1.1700 & 2.4700 & 1.4270 \\
\hline \multirow{3}{*}{1.50} & 0.01 & 1.0863 & 1.1990 & 1.2120 & 1.1420 & 1.1670 & 1.3930 & 1.3610 & 1.1220 \\
\hline & 0.05 & 1.2180 & 1.9920 & 1.1090 & 1.0745 & 1.0860 & 1.1530 & 2.3600 & 1.3110 \\
\hline & 0.10 & 1.3690 & 4.4800 & 1.1100 & 1.0695 & 1.0828 & 1.1600 & 2.1800 & 1.4960 \\
\hline
\end{tabular}


Table 8. $R E\left(\hat{\theta}_{4}, Y_{1}\right)$ when $\lambda=2.5$.

\begin{tabular}{|c|c|c|c|c|c|c|c|c|c|}
\hline \multirow[b]{2}{*}{$c$} & \multirow[b]{2}{*}{$\alpha$} & \multicolumn{8}{|c|}{$\delta$} \\
\hline & & 0.4 & 0.6 & 0.8 & 1.0 & 1.2 & 1.4 & 1.6 & 1.8 \\
\hline \multicolumn{10}{|c|}{$r=6$} \\
\hline \multirow{4}{*}{0.25} & 0.01 & 1.0267 & 1.0065 & 1.0053 & 1.0058 & 1.0069 & 1.0086 & 1.0117 & 1.0177 \\
\hline & 0.05 & 1.0578 & 1.0092 & 1.0058 & 1.0060 & 1.0075 & 1.0105 & 1.0171 & 1.0330 \\
\hline & 0.10 & 1.0904 & 1.0118 & 1.0064 & 1.0063 & 1.0082 & 1.0127 & 1.0234 & 1.0533 \\
\hline & 0.01 & 1.2480 & 1.6250 & 1.0662 & 1.0335 & 1.0243 & 1.0202 & 1.0187 & 1.0192 \\
\hline \multirow{3}{*}{0.50} & 0.05 & 1.4060 & 1.3910 & 1.0831 & 1.0351 & 1.0255 & 1.0229 & 1.0242 & 1.0294 \\
\hline & 0.10 & 1.5160 & 1.3740 & 1.1010 & 1.0372 & 1.0272 & 1.0261 & 1.0305 & 1.0421 \\
\hline & 0.01 & 2.3400 & 1.0675 & 1.0327 & 1.0255 & 1.0227 & 1.0218 & 1.0229 & 1.0265 \\
\hline \multirow{3}{*}{1.00} & 0.05 & 2.6100 & 1.1060 & 1.0375 & 1.0266 & 1.0242 & 1.0254 & 1.0305 & 1.0424 \\
\hline & 0.10 & 2.7500 & 1.1450 & 1.0424 & 1.0282 & 1.0261 & 1.0295 & 1.0393 & 1.0621 \\
\hline & 0.01 & 2.4200 & 1.0817 & 1.0416 & 1.0332 & 1.0303 & 1.0302 & 1.0338 & 1.0441 \\
\hline \multirow{2}{*}{1.50} & 0.05 & 2.7100 & 1.1260 & 1.0474 & 1.0347 & 1.0325 & 1.0355 & 1.0456 & 1.0716 \\
\hline & 0.10 & 2.7900 & 1.1720 & 1.0534 & 1.0367 & 1.0352 & 1.0414 & 1.0593 & 1.1070 \\
\hline \multicolumn{10}{|c|}{$r=8$} \\
\hline \multirow{4}{*}{0.25} & 0.01 & 1.0478 & 1.0060 & 1.0042 & 1.0045 & 1.0054 & 1.0071 & 1.0106 & 1.0188 \\
\hline & 0.05 & 1.1200 & 1.0094 & 1.0047 & 1.0047 & 1.0060 & 1.0092 & 1.0174 & 1.0428 \\
\hline & 0.10 & 1.1960 & 1.0126 & 1.0052 & 1.0050 & 1.0067 & 1.0115 & 1.0258 & 1.0776 \\
\hline & 0.01 & 1.3780 & 1.9530 & 1.0462 & 1.0239 & 1.0177 & 1.0152 & 1.0150 & 1.0171 \\
\hline \multirow{3}{*}{0.50} & 0.05 & 1.6060 & 1.4730 & 1.0586 & 1.0250 & 1.0188 & 1.0181 & 1.0216 & 1.0317 \\
\hline & 0.10 & 1.7270 & 1.4480 & 1.0711 & 1.0265 & 1.0203 & 1.0215 & 1.0294 & 1.0510 \\
\hline & 0.01 & 2.9300 & 1.0607 & 1.0247 & 1.0190 & 1.0170 & 1.0168 & 1.0187 & 1.0243 \\
\hline \multirow{3}{*}{1.00} & 0.05 & 2.5000 & 1.1050 & 1.0289 & 1.0198 & 1.0184 & 1.0206 & 1.0279 & 1.0467 \\
\hline & 0.10 & 2.2200 & 1.1520 & 1.0332 & 1.0209 & 1.0201 & 1.0248 & 1.0388 & 1.0773 \\
\hline & 0.01 & 2.9100 & 1.0737 & 1.0315 & 1.0248 & 1.0227 & 1.0233 & 1.0277 & 1.0403 \\
\hline \multirow{2}{*}{1.50} & 0.05 & 2.5000 & 1.1260 & 1.0367 & 1.0259 & 1.0247 & 1.0288 & 1.0418 & 1.0793 \\
\hline & 0.10 & 2.2200 & 1.1830 & 1.0419 & 1.0273 & 1.0271 & 1.0349 & 1.0587 & 1.1350 \\
\hline \multicolumn{10}{|c|}{$r=10$} \\
\hline \multirow{4}{*}{0.25} & 0.01 & 1.0934 & 1.0060 & 1.0034 & 1.0037 & 1.0045 & 1.0062 & 1.0102 & 1.0216 \\
\hline & 0.05 & 1.2490 & 1.0102 & 1.0040 & 1.0039 & 1.0051 & 1.0085 & 1.0189 & 1.0596 \\
\hline & 0.10 & 1.3940 & 1.0145 & 1.0045 & 1.0041 & 1.0057 & 1.0110 & 1.0300 & 1.1180 \\
\hline & 0.01 & 1.5560 & 2.3000 & 1.0359 & 1.0186 & 1.0139 & 1.0124 & 1.0132 & 1.0173 \\
\hline \multirow{2}{*}{0.50} & 0.05 & 1.7900 & 1.5490 & 1.0462 & 1.0194 & 1.0150 & 1.0156 & 1.0214 & 1.0387 \\
\hline & 0.10 & 1.8780 & 1.5220 & 1.0566 & 1.0205 & 1.0164 & 1.0192 & 1.0312 & 1.0698 \\
\hline \multirow{4}{*}{1.00} & 0.01 & 2.9000 & 1.0596 & 1.0200 & 1.0151 & 1.0136 & 1.0139 & 1.0168 & 1.0249 \\
\hline & 0.05 & 2.1600 & 1.1130 & 1.0240 & 1.0157 & 1.0150 & 1.0180 & 1.0281 & 1.0580 \\
\hline & 0.10 & 2.0700 & 1.1750 & 1.0279 & 1.0166 & 1.0165 & 1.0225 & 1.0419 & 1.1080 \\
\hline & 0.01 & 2.8500 & 1.0726 & 1.0256 & 1.0198 & 1.0183 & 1.0194 & 1.0249 & 1.0415 \\
\hline \multirow{2}{*}{1.50} & 0.05 & 2.1600 & 1.1370 & 1.0304 & 1.0206 & 1.0201 & 1.0252 & 1.0422 & 1.0997 \\
\hline & 0.10 & 2.0700 & 1.2130 & 1.0353 & 1.0218 & 1.0223 & 1.0317 & 1.0637 & 1.1940 \\
\hline
\end{tabular}


than the underestimation.

\subsection{When $\lambda=0.5$}

From these tables it is observed that the shrinkage testimators $\hat{\theta}_{4}$ perform better than the improved estimator $Y_{1}$ for all considered values of $r, c, \delta, \lambda$ and $\alpha$. The testimators $\hat{\theta}_{1}, \hat{\theta}_{2}$ and $\hat{\theta}_{3}$ perform better than $Y_{1}$ when $c \geq 0.5$. The testimators $\hat{\theta}_{4}$ attain maximum efficiency at the point $\delta=0.4$ and others near to the point $\delta=$ 1.

For fixed $c$ and level of significance $\alpha$, as the uncensored sample size $r$ increases, the relative efficiency decreases in all considered values of $\delta$ for all the testimators.

For fixed $r$ and $\alpha$, when $c$ increases the relative efficiency increases in all considered values of $\delta$ for all testimators.

It has been seen that as the level of significance $\alpha$ increases the relative efficiency increases in all considered values of $\delta$ for all testimators.

\subsection{When $\lambda=2.5$}

From these tables it is observed that the shrinkage testimators $\hat{\theta}_{4}$ perform better than the improved estimator $Y_{1}$ for all considered values of $r, c, \delta, \lambda$ and $\alpha$. The testimators $\hat{\theta}_{1}, \hat{\theta}_{2}$ and $\hat{\theta}_{3}$ perform better than $Y_{1}$ when $c \geq 0.5$. The testimators $\hat{\theta}_{4}$ attain maximum efficiency at the point $\delta=0.4$ and others near to the point $\delta=1.6$.

For fixed $c$ and level of significance $\alpha$, as the uncensored sample size $r$ increases, the relative efficiency decreases in the region $0.8 \leq \delta \leq 1.6$ for the testimator $\hat{\theta}_{1}$, and in the region $0.8 \leq \delta \leq 1.4$ for the testimators $\hat{\theta}_{2}$ and $\hat{\theta}_{3}$, and also for testimator $\hat{\theta}_{4}$ it decreases for all considered values of $\delta$.

For fixed $r$ and $\alpha$, when $c$ increases the relative efficiency increases in all considered values of $\delta$ for all testimators.

It has been seen that as the level of significance $\alpha$ increases the relative efficiency increases in $1.6 \leq \delta \leq 0.6$ and also in $0.8 \leq \delta \leq 1.4$ when $c=0.25,0.5$, and decreases for $0.8 \leq \delta \leq 1.4$ when $c \geq 1.5$ and $r \geq 8$ for testimators $\hat{\theta}_{1}, \hat{\theta}_{2}$ and $\hat{\theta}_{3}$, and for testimator $\hat{\theta}_{4}$ it increases for all considered values of $\delta$.

\section{Recommendations}

In this study, some shrinkage testimators $\left(\hat{\theta}_{1}, \hat{\theta}_{2}, \hat{\theta}_{3}\right.$ and $\hat{\theta}_{4}$ ) for the scale parameter of a gamma distribution, when type-II censored data are available, suggested that under the ILL loss function assuming the shape parameter was to be known. The comparisons of the proposed testimators made with improved estimator $Y_{1}$. The recommendations have been presented, based on the relative efficiency for all the shrinkage testimator. From the previous observations, the shrinkage testimators $\hat{\theta}_{4}$ perform better than the improved estimator $Y_{1}$ for all considered values of $r, c, \delta, \lambda$ and $\alpha$, and the testimators $\hat{\theta}_{1}, \hat{\theta}_{2}$ and $\hat{\theta}_{3}$ perform better than $Y_{1}$ when $c \geq 0.5$. Since the shrinkage testimators $\hat{\theta}_{4}$ always perform better than other shrinkage testimators if the gain in efficiency does not matter, therefore we strongly suggest using the shrinkage testimators $\hat{\theta}_{4}$ for the scale parameter of a gamma distribution, when Type-II censored data are available, suggested under the ILL loss function.

\section{Acknowledgements}

The authors would like to thank the referee and the editor for a careful reading of the paper and for valuable comments which improved the presentation of the paper. The second author gratefully appreciates his wife, Dr. Saemeh Dehghan, who kindly helps the researcher in the study.

\section{REFERENCES}

[1] A. Parsian and S. N. U. A. Kirmani, "Estimation under LINEX loss function,” In: A. Ulla, Ed., Handbook of Applied Econometrics and Statistical Inference, 165 Dekker, New York, 2002, pp. 53-76.

[2] H. Pazira and A. Shadrokh, "Comparison of LINEX and Precautionary Bayes Estimators on the Gamma Distribution Using Censored Data,” Journal of Statistics and Management Systems, Vol. 14, No. 3, 2011, pp. 617-638. doi:10.1080/09720510.2011.10701575

[3] T. S. Ferguson, "Mathematical Statistics: A Decision Theoretic Approach,” Academic Press, New York, 1967.

[4] A. Zellner and M.S. Geisel, "Sensitivity of Control to Uncertainty and Form of the Criterion Function,” In the future of statistics, Ed Donald G. Watts, Academic Press, New York, 1968, pp. 269-289.

[5] J. Aitchison and I. R. Dunsmore, "Statistical Prediction Analysis,” Cambridge University Press, Cambridge, 1975. doi:10.1017/CBO9780511569647

[6] H. R. Varian, "A Bayesian Approach to Real Estate Assessment,” In: L. J. Savage, S. E. Feinberg and A. Zellner, Eds., Studies in Bayesian Econometrics and Statistics: In Honor of L. J. Savage, North-Holland Pub. Co., Amsterdam, 1975, pp. 195-208.

[7] J. O. Berger, "Statistical Decision Theory-Foundation Concepts and Methods," Springer-Verlag, New York, 1980. doi:10.1007/978-1-4757-1727-3

[8] A. P. Basu and N. Ebrahimi, "Bayesian Approach to Life Testing and Reliability Estimation Using Asymmetric Loss Function," Journal of Statistical Planning and Inferences, Vol. 29, No. 1-2, 1991, pp. 21-31. doi:10.1016/0378-3758(92)90118-C

[9] B. N. Pandey, "Testimator of the Scale Parameter of the Exponential Distribution Using LINEX Loss Function,” Communication in Statistics-Theory and Methods, Vol. 
26, No. 6, 1997, pp. 2191-2202. doi:10.1016/0026-2714(88)90294-6

[10] A. A. Soliman, "Comparison of Linex and QuadraticBayes Estimators Foe the Rayleigh Distribution," Communication in Statistics-Theory and Methods, Vol. 29, No. 1, 2000, pp. 95-107. doi:10.1080/03610920008832471

[11] G. Prakash and D. C. Singh, "Shrinkage Estimation in Exponential Type-II Censored Data under LINEX Loss," Journal of the Korean Statistical Society, Vol. 37, No. 1, 2008, pp. 53-61. doi:10.1016/j.jkss.2007.07.002

[12] A. Parsian and N. S. Farsipour, "Estimation of the Mean of the Selected Population under Asymmetric Loss Function,” Metrika, Vol. 50, No. 2, 1999, pp. 89-107.

[13] U. Singh, P. K. Gupta and S. K. Upadhyay, "Estimation of Exponentiated Weibull Shape Parameters under LINEX Loss Function," Communication in Statistics-Simulation, Vol. 31, No. 4, 2002, pp. 523-537. doi:10.1081/SAC-120004310

[14] N. Misra and E. V. D. Meulen, "On Estimating the Mean of the Selected Normal Population under the LINEX Loss Function,” Metrika, Vol. 26, No. 9, 2003, pp. 173-184. doi:10.1080/03610929708832041

[15] J. Ahmadi, M. Doostparast and A. Parsian, "Estimation and Prediction in a Two-Parameter Exponential Distribution Based on K-Record Values under LINEX Loss Function," Communications in Statistics-Theory and Methods, Vol. 34, No. 4, 2005, pp. 795-805. doi:10.1081/STA-200054393

[16] Y. Xiao, Y. Takada and N. Shi, "Minimax Confidence Bound of the Normal Mean under an Asymmetric Loss Function,” Annals of Statistical Mathematics, Vol. 57, No.

\section{1, 2005, pp. 167-182. doi:10.1007/BF02506886}

[17] D. C. Singh, G. Prakash and P. Singh, "Shrinkage Testimator for the Shape Parameter of Pareto Distribution Using LINEX Loss Function," Communication in Statistics-Theory and Methods, Vol. 36, No. 4, 2007, pp. 741753. doi:10.1080/03610920601033694

[18] A. K. Rao and R. S. Srivastava, "Bayesian Estimation of the Scale Parameter of Gamma Distribution under Linex Loss Function with Censoring,” In: B. N. Pandey, Ed., Statistical Techniques in Life-Testing, Reliability, Sampling Theory and Quality Control, Narosa Pub House, Daryaganj, 2002, pp. 287-295.

[19] J. R. Thompson, "Some Shrunken Techniques for Estimateing the Mean," Journal of the American Statistical Association, Vol. 63, No. 321, 1968, pp. 113-122. doi:10.2307/2283832

[20] V. B. Waikar, F. J. Schuurmann and T. E. Raghunathan, "On a Two Stage Shrunken Testimator of the Mean of a Normal Distribution," Communications in Statistics-Theory and Methods, Vol. 13, No. 15, 1984, pp. 1901-1913. doi:10.1080/03610928408828802

[21] S. R. Adke, V. B. Waikar and F. J. Schuurmann, “A Two Stage Shrinkage Testimator for the Mean of an Exponential Distribution," Communication in Statistics-Theory and Methods, Vol. 16, No. 6, 1987, pp. 1821-1834. doi:10.1080/03610928708829474

[22] B. N., Pandey, H. J. Malik and R. Srivastava, "Shrinkage Testimator for the Variance of a Normal Distribution at Single and Double Stages," Microelectron Reliability, Vol. 28, No. 6, 1988, pp. 929-944. doi:10.1016/0026-2714(88)90294-6 